\title{
Análise da dinâmica populacional entre a broca da cana-de-açúcar e seus parasitoides do estágio do ovo e larval com variação sazonal
}

\author{
Daiane Frighetto Frighetto ${ }^{1}$ \\ PPGMAp/UFRGS, Porto Alegre, RS \\ João Inácio Moreira Bezerra² \\ IFM/UFPEL, Pelotas, RS \\ Alexandre Molter ${ }^{3}$ \\ IFM/UFPel, Pelotas, RS \\ Marat Rafikov ${ }^{4}$ \\ CECS/UFABC, Santo André, SP
}

\begin{abstract}
Resumo. A produção de cana-de-açúcar no Brasil é um importante setor do agronegócio. Ela é matéria-prima para a produção de biocombistíveis, bioplásticos, bioenergia, bebidas e alimentos. Nas últimas décadas, com a expansão das plantações e a proibição das queimadas, houve um aumento significativo na população de pragas, sendo a broca da cana-de-açúcar, Diatraea saccharalis, a principal delas. Essa praga cava galerias internas nos colmos das plantas causando prejuízos, diminuindo a qualidade e o rendimento industrial. Dessa forma, o propósito deste trabalho é estudar a interação entre a broca da cana-de-açúcar, primeiramente, com seu parasitoide larval, Cotesia flavipes e na sequência, incluindo também o seu parasitoide do ovo, Trichogramma galloi, que demonstram desempenho satisfatório no controle biológico da broca da cana-de-açúcar. A interação entre essas populações é modelada por sistemas do tipo parasitoide-hospedeiro, para o qual é feito o estudo considerando os parâmetros constantes e também com a variação sazonal. As simulações mostram que a inclusão da variação sazonal nos dois sistemas altera a dinâmica, podendo gerar comportamento caótico.
\end{abstract}

Palavras-chave. Broca da cana-de-açúcar, Cotesia flavipes, Trichogramma galloi, Sazonalidade.

\section{Introdução}

Apesar da facilidade de adaptação ao clima do Brasil, a cultura da cana-de-açúcar enfrenta uma série de problemas fitossanitários, tais como a incidência de insetos-praga. Uma das principais pragas que atacam a cultura de cana-de-açúcar é a broca-da-cana (Diatraea saccharalis), um inseto que penetra no interior da planta e cava galerias internas, causando grandes prejuízos aos produtores. Sabemos, por exemplo, que cada $1 \%$ da infestação de plantas resulta em $0,2 \%$ de perdas na produção de etanol.

O controle biológico, através dos parasitoides da larva do ovo, é a uma alternativa viável para o controle da larva da broca, uma vez que ela fica dentro do colmo da planta e a pulverização

\footnotetext{
${ }^{1}$ daiane.frighetto@gmail.com.br

2 jimbezerra@inf.ufpel.edu.br

3 alexandre.molter@ufpel.edu.br

4marat.rafikov@ufabc.edu.br
} 
de inseticidas, nesse caso, é inecifiente. Na área de 1.000.000 hectares que hoje é controlada biologicamente, o rendimento diário é de 2.5000 .000 litros de etanol [2].

No entanto, o controle biológico continua sendo um grande desafio na produção de cana-deaçúcar. Apesar de que o controle biológico da praga Diatraea saccharalis pelo parasitoide Cotesia flavipes é considerado um sucesso no Brasil, segundo [2], ainda há áreas onde esse parasitoide não controla bem. Desta forma, muitos estudos apontam que, para um controle biológico mais eficiente, a liberação de Cotesia flavipes, associada ao uso do parasitoide do ovo, Trichogramma galloi, é considerada uma opção interessante e promissora $[2,4,7]$.

Em sistemas populacionais com mais que duas espécies, a dinâmica das populações praga parasitoides torna-se complexa, e consequentemente fica mais difícil prever invasões dessa praga. Para entender essas interações, a modelagem matemática tem papel importante na tomada de decisões sobre o controle da população de pragas e contribui com informações sobre a estabilidade de sistemas naturais [3], que, juntamente com simulações computacionais, ajudam a desvendar tais sistemas complexos e estudar como pragas interagem com outros organismos no meio ambiente. Por isso, o objetivo deste trabalho é investigar interações entre broca da cana-de-açúcar e seus parasitoides no meio ambiente com variação sazonal.

\section{Modelagem matemática e simulações da interação Broca da cana com o parasitoide da larva (Cotesia flavipes) en- volvendo sazonalidade}

Um modelo de interação entre broca da cana-de-açúcar e seu parasitoide de larvas foi proposto em [7]. No trabalho [1], este modelo foi investigado no meio ambiente com variação sazonal e foi mostrado que a variação sazonal pode gerar regimes caóticos e, de forma brusca, diminuir a população de parasitoides. O modelo matemático de interação entre broca da cana-de-açúcar e seu parasitoide de larvas tem a seguinte forma [7]:

$$
\left\{\begin{array}{l}
\dot{H}=r\left(1-\frac{H}{K}\right) H-n_{1} H-\beta P H, \\
\dot{I}=\beta H P-I\left(m_{2}+n_{2}\right), \\
\dot{P}=\delta n_{2} I-m_{3} P
\end{array}\right.
$$

onde $H$ é a densidade larval da broca da cana-de-açúcar (Diatraea saccharalis), $I$ é a densidade da população de $H$ que é parasitada por $P$, enquanto $P$ é a densidade da larva parasita(Cotesia flavipes). Quanto aos parâmetros, tem-se que $r$ representa a taxa de crescimento intrínseco da praga; $K$ é a capacidade de suporte do ambiente; $m_{2}$ e $m_{3}$ são as taxas de mortalidade de $I$ e $P$, respectivamente; $n_{1}$ é a fração da população da larva que se transforma em pupa em um instante de tempo $t ; n_{2}$ é a fração da larva parasitada da qual parasitoides adultos emergem em um instante de tempo $t ; \beta$ é a taxa intrínseca de parasitismo; $\delta$ é o número de parasitoides adultos que emergem de uma larva parasitada em um instante $t$.

Esse modelo com coeficientes constantes não leva em conta que nos meses do inverno o estágio de pupa pode durar muito tempo, passando por um período chamado de "dormência". No trabalho [1] foi feito um estudo numérico da variação sazonal da taxa de crescimento representada pelo parâmetro $r$. Esse parâmetro tem seus valores definidos pela seguinte função:

$$
r=0,19\left(1+r_{1} \operatorname{sen}\left(\frac{2 \pi t}{365}\right)\right),
$$

onde $r_{1} \in[0,1]$ representa a intensidade da sazonalidade. Um valor de $r_{1}=0$ representa a ausência de sazonalidade, enquanto $r_{1}=1$ implica que o valor máximo de $r$ é o dobro de seu valor médio. 
A escollha de (2) em função da função seno se deve a manutenção inicial da taxa de crescimento intrínseco.

As simulações mostraram que a sazonalidade pode causar mudanças drásticas na dinâmica do agroecossistema. O sistema (1), que não possui sazonalidade, apresenta um atrator do tipo ciclo limite (Figura 1-a). Já o sistema com sazonalidade mostra um atrator estranho para o valor de $r_{1}=0,8$ (Figura 1-b). O diagrama de bifurcação na Figura 2 confirma que para valores do coeficiente $r_{1}$ maiores que 0,7 , o sistema apresenta o comportamento caótico. As simulações foram realizadas com os seguintes valores dos parâmetros: $r=0,19 ; n_{1}=1 / 50 ; \beta=0,0000083$; $m_{2}=0,00257 ; n_{2}=1 / 16 ; m_{3}=0,2$ e $\gamma=40$, conforme [7].

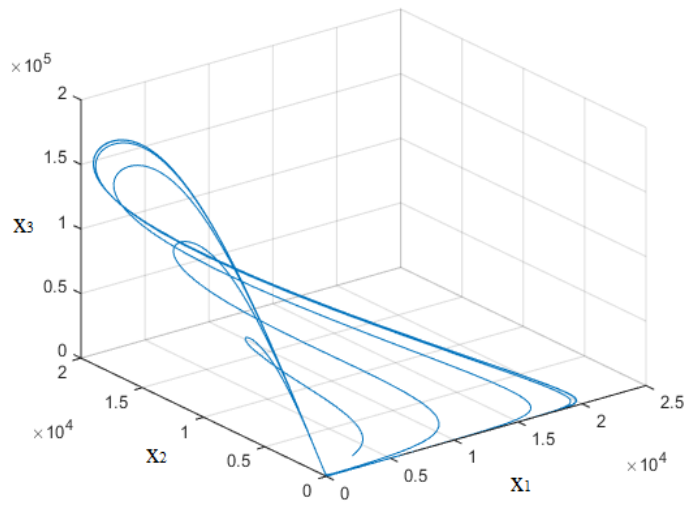

(a)

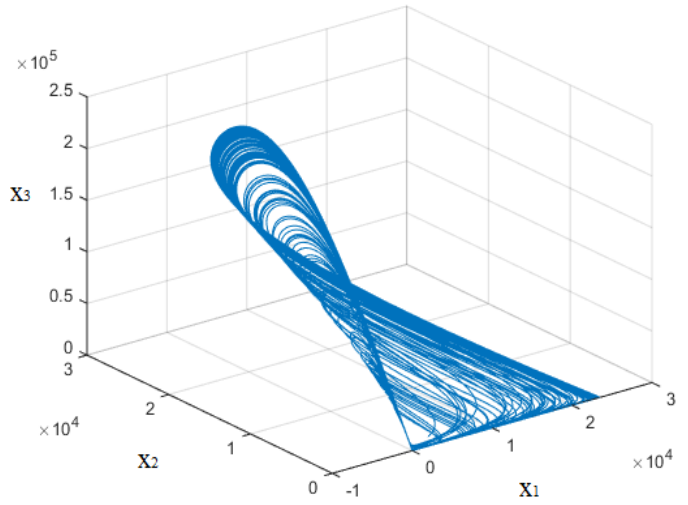

(b)

Figura 1: a) Retrato de fase do sistema (1) sem considerar a sazonalidade. b) Retrato de fase do sistema (1) considerando a sazonalidade.

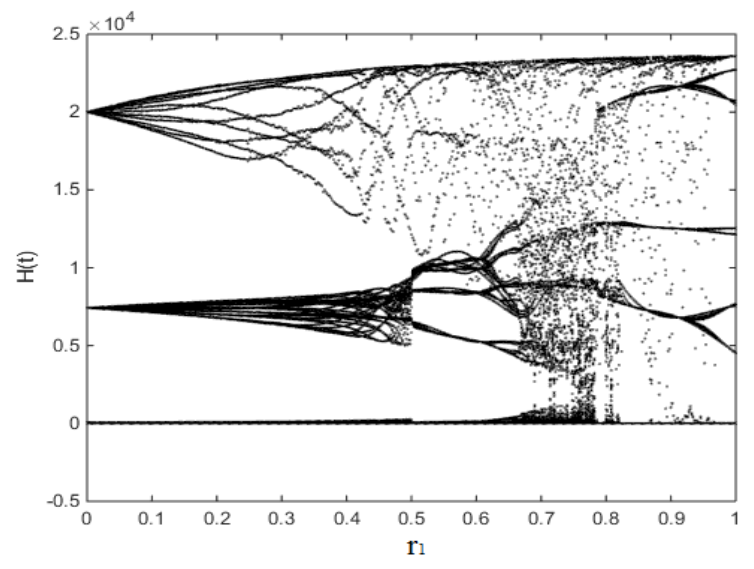

Figura 2: Diagrama de bifurcação do sistema (1), de $H(t)$, com sazonalidade.

Pode-se observar pela Figura 2 que o sistema 1, incluindo a sazonalidade, muda completamente seu comportamento, indo de ciclo limite para uma dinâmica caótica. 


\section{Modelagem matemática e simulações da interação Broca da cana com os parasitoides da larva (Cotesia flavipes) e do ovo (Trichogramma galloi) com sazonalidade}

O aparecimento de regimes caóticos pode explicar os casos quando o parasitoide das larvas Cotesia flavipes não controla bem a praga. Nessas situações, os especialistas do controle biológico recomendam o uso simultâneo do parasitoide das larvas, Cotesia flavipes, e dos ovos, Trichogramma galloi. No trabalho [6], foi proposto um modelo de interação de larvas e ovos da Diatraea saccharalis com seus parasitoides. Esse mesmo modelo foi usado em [4] para investigar algumas estratégias do controle biológico, contudo, não foram consideradas as populações de ovos e larvas parasitadas. Desse modo, para investigar a interação da população de praga (em estágios de ovos, ovos parasitados, larvas e larvas parasitadas) com as populações de parasitoide de ovos e das larvas, consideraremos o seguinte modelo:

$$
\left\{\begin{array}{l}
\dot{x_{1}}=r x_{1}\left(1-\frac{x_{1}}{K}\right)-m_{1} x_{1}-n_{1} x_{1}-\alpha x_{1} x_{3}, \\
\dot{x_{2}}=\alpha x_{1} x_{3}-m_{2} x_{2}-n_{2} x_{2}, \\
\dot{x_{3}}=\gamma_{1} n_{2} x_{2}-m_{3} x_{3} \\
\dot{x_{4}}=n_{1} x_{1}-m_{4} x_{4}-n_{3} x_{4}-\beta x_{4} x_{6}, \\
\dot{x_{5}}=\beta x_{4} x_{6}-m_{5} x_{5}-n_{4} x_{5}, \\
\dot{x_{6}}=\gamma_{2} n_{4} x_{5}-m_{6} x_{6},
\end{array}\right.
$$

onde $x_{1}, x_{2}, x_{3}, x_{4}, x_{5}, x_{6}$ representam, respectivamente, as densidades de ovos normais da broca da cana, de ovos parasitados, de adultos de parasitoides de ovos (Trichogramma galloi), de larvas, de larvas parasitadas e de adultos de parasitoides de larvas (Cotesia flavipes). Os coeficientes do sistema de equações têm os seguintes significados biológicos: $K$ é capacidade de suporte do ambiente; $r$ é o coeficiente que caracteriza a taxa de reposição de ovos; $n_{1}$ é coeficiente que caracteriza a parte dos ovos normais que se tornaram larvas, no instante $t ; n_{2}$ é coeficiente que caracteriza a parte de ovos parasitados de quais emergiram parasitoides de ovos, no instante $t$; $n_{3}$ é coeficiente que caracteriza a parte de larvas que se tornaram pupas; $n_{4}$ é coeficiente que caracteriza a parte de larvas parasitadas de quais emergiram parasitoides de larvas, no instante $t ; m_{1}$ é coeficiente de mortalidade dos ovos normais; $m_{2}$ é coeficiente de mortalidade dos ovos parasitados; $m_{3}$ é coeficiente de mortalidade dos adultos do parasitoide de ovos; $m_{4}$ é coeficiente de mortalidade das larvas; $m_{5}$ é coeficiente de mortalidade das larvas parasitadas; $m_{6}$ é coeficiente de mortalidade dos adultos do parasitoide de larvas; $\alpha$ é coeficiente que caracteriza a taxa de parasitismo de ovos; $\beta$ é coeficiente que caracteriza a taxa de parasitismo de larvas; $\gamma_{1}$ é número de parasitoides emergidos de um ovo parasitado; $\gamma_{2}$ é número de parasitoides emergidos de uma larva parasitada.

Para investigar a dinâmica do sistema (3) foram usados os seguintes valores de taxas de parasitismo: $\alpha=0,0001723$ e $\beta=0,0000083$ conforme [5,7], respectivamente. Os valores dos outros coeficientes do modelo (3) foram adaptados com base nos valores médios do ciclo de vida, mortalidade e outras informações sobre biologia das espécies: $K=25000 ; r=0,19 ; n_{1}=1 / 8 ; n_{2}=1 / 9$; $n_{3}=1 / 50 ; n_{4}=1 / 16 ; m_{1}=0 ; m_{2}=0,03566 ; m_{3}=1 / 4 ; m_{4}=m_{5}=0,00257 ; m_{6}=1 / 5$; $\gamma_{1}=2,29$ e $\gamma_{2}=40$ [7]. Os resultados das simulações numéricas do modelo (3), sem sazonalidade, estão na Figura 3, que nos mostram que todas as espécies coexistem em uma dinâmica determinada por um simples ciclo limite.

Para um estudo numérico da dinâmica do sistema (3), com variação sazonal, a taxa de crescimento representada pelo parâmetro $r$ foi considerada da forma (2). Nesse caso, o diagrama de bifurcação do modelo (3), com sazonalidade, está representado na Figura 4. Nele podemos ver 
várias regiões exibindo caos e "janelas" que correspondem aos ciclos limite.

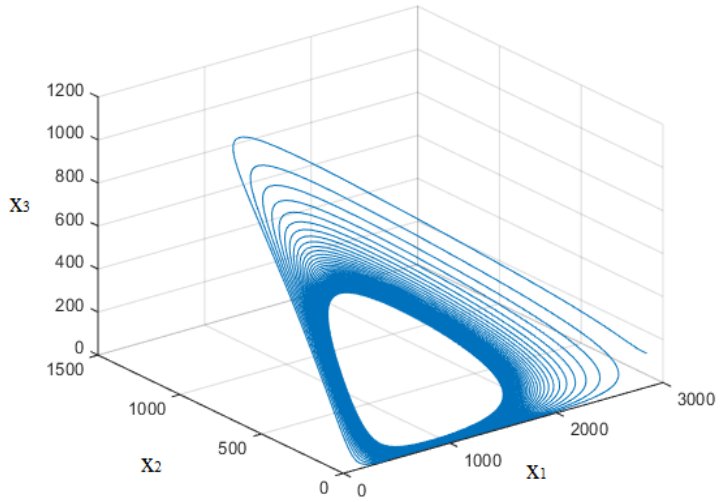

(a)

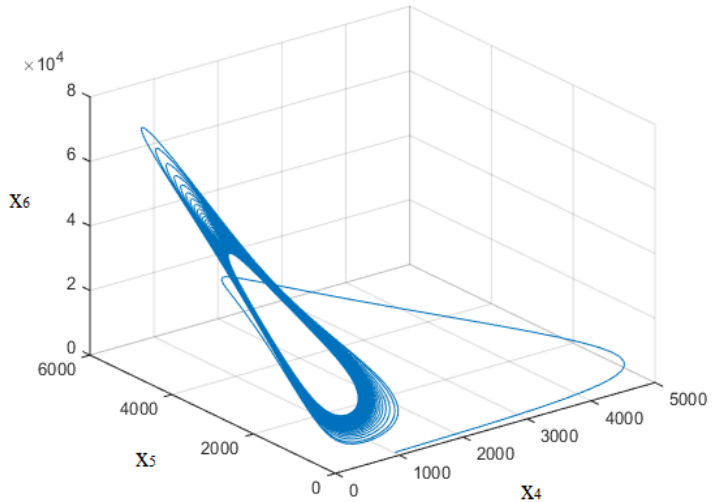

(b)

Figura 3: Resultados de simulações numéricas do modelo (3) sem sazonalidade.

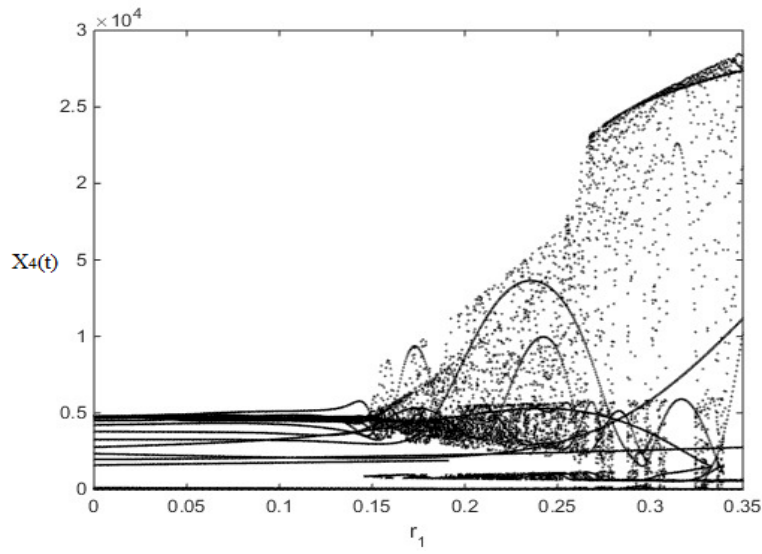

Figura 4: Diagrama de bifurcação do sistema (3), população $x_{4}$, com sazonalidade.

Diferentemente do sistema envolvendo somente um parasitoide, a Cotesia flavipes, o sistema (3) começa a apresentar regimes caóticos ainda para pequenas mudanças sazonais (a partir do valor $\left.r_{1}=0,15\right)$. Atratores caóticos do sistema (3) para o valor do parâmetro $r_{1}=0,2$ são apresentados na Figura 5:(a-b), enquanto que na Figura 5:(c-d) aparecem ciclos limites múltiplos, que correspondem a "janela" com valor de $r_{1}=0,33$.

Para considerar as consequências da dinâmica caótica do sistema (3) na incidência da broca da cana-de-açúcar, durante o período de um ano e meio, foram realizadas simulações numéricas para diferentes valores iniciais. Comparando a densidade de broca da cana-de-açúcar (a variável $x_{4}$ ) na Figura 5:(e-f) mostram que, até para densidades iniciais menores que 2500, existe uma tendência de crescimento da população de pragas. Isto significa que a única aplicação do controle no momento inicial, usando dois parasitoides, pode levar a um resultado não satisfatório. Assim, precisamos de uma estratégia adequada durante este período. 


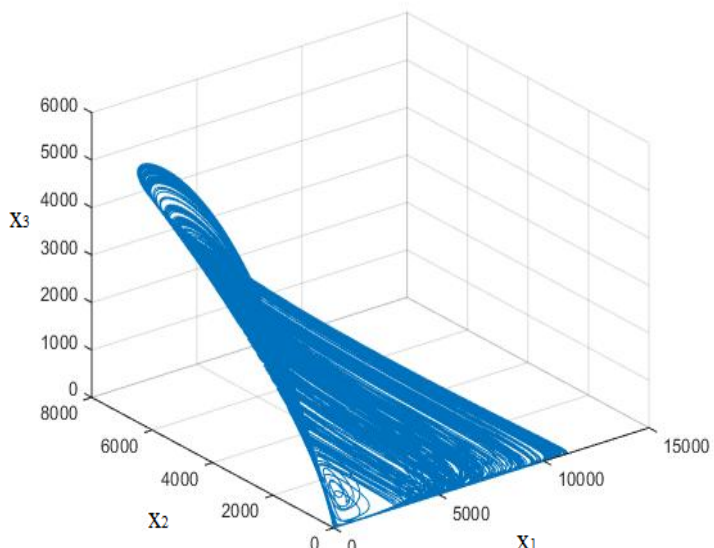

(a)

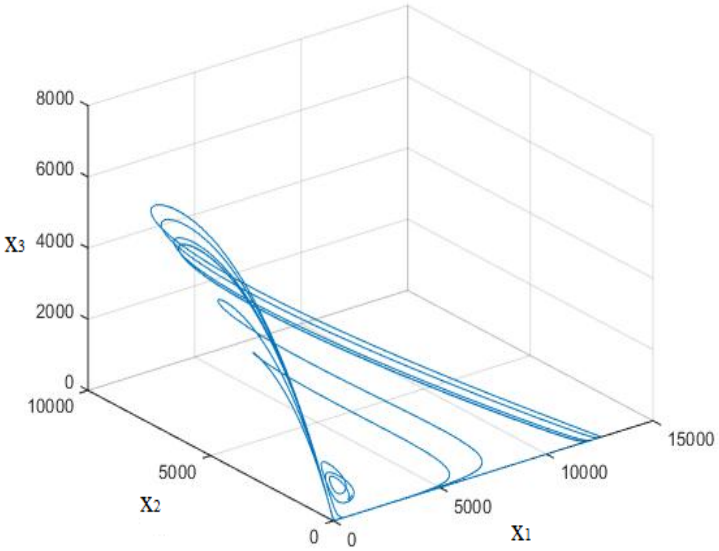

(c)

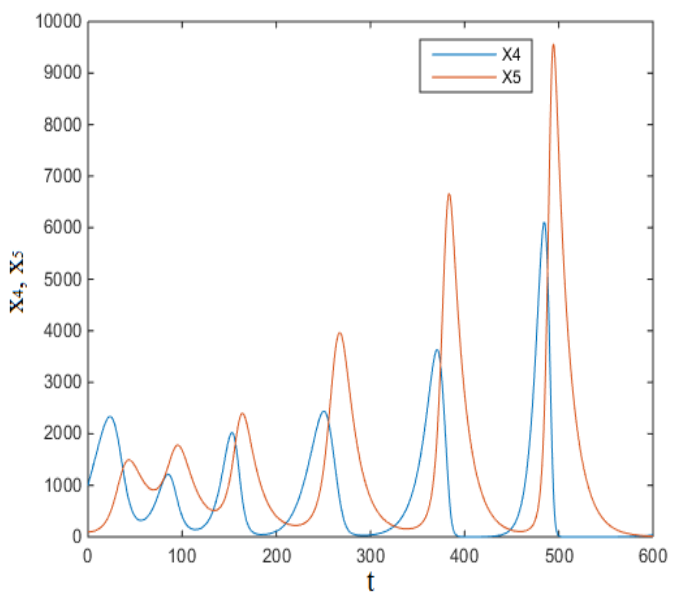

(e)

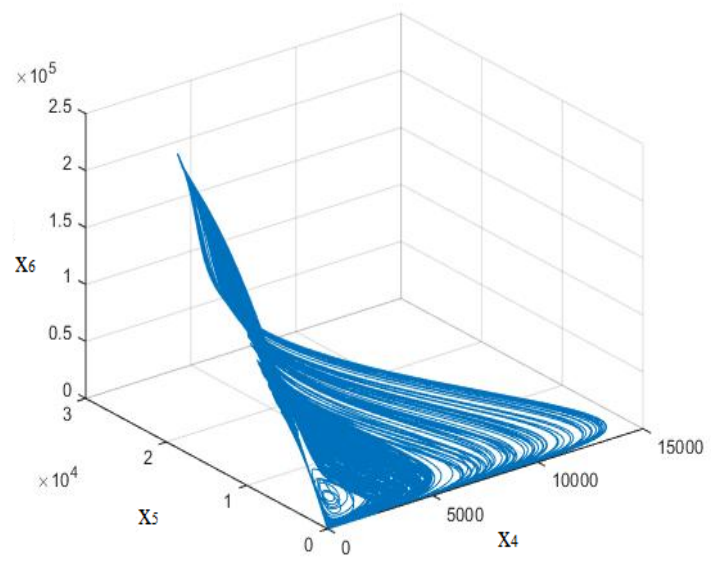

(b)

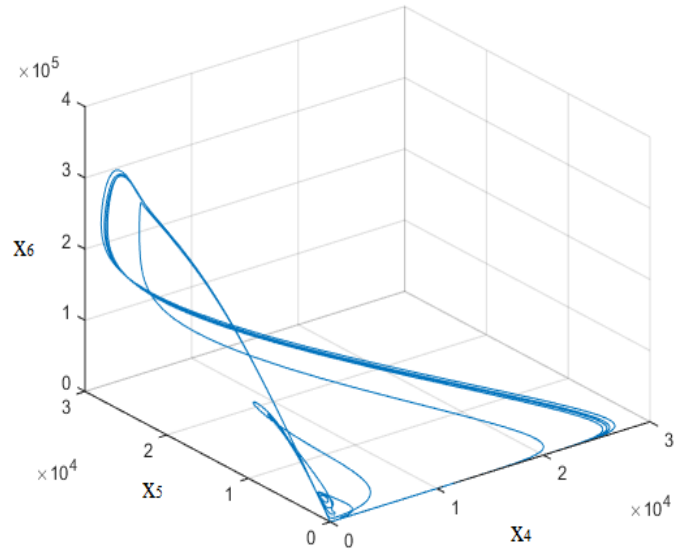

(d)

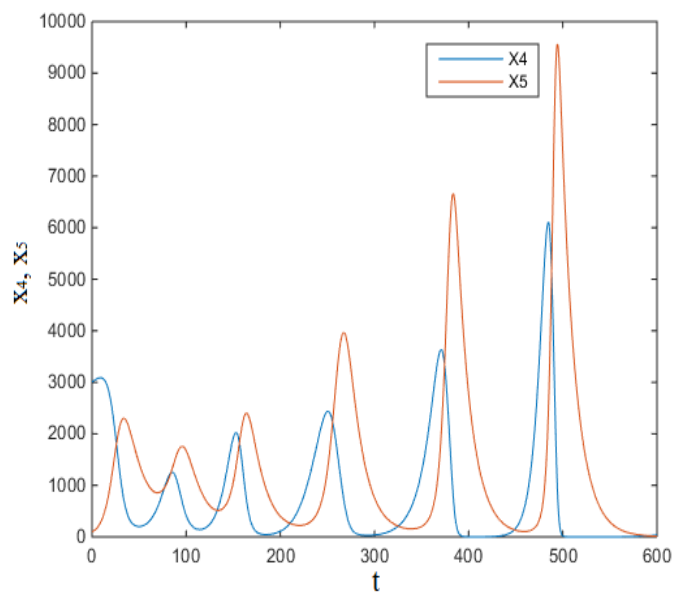

(f)

Figura 5: a) e b) Atratores caóticos do sistema (3) para valor do parâmetro $r_{1}=0,2$; c) e d) Atratores do tipo ciclo limite do sistema (3) para o valor do parâmetro $r_{1}=0,33$; e) Densidades de larvas e larvas parasitadas para condição inicial $x_{4}=1000$ larvas; e f) Densidades de larvas e larvas parasitadas para condição inicial $x_{4}=3000$ larvas. 
Pode-se observar pelas Figuras 3, 4 e 5 que o sistema 3, incluindo a sazonalidade, também altera seu comportamento, indo de ciclo limite para uma dinâmica caótica. Só que neste caso, a influência da sazonalidade já aparece nos primeiros valores do coeficiente $r_{1}$, tomados nas simulações.

\section{Conclusões}

Neste trabalho primeiramente trazemos a influência da sazonalidade na dinâmica de interações entre a broca da cana-de-açúcar (Diatraea saccharalis) e seu parasitoide larval (Cotesia flavipes), baseado no modelo proposto em [7]. O estudo mostrou que o sistema (1), com sazonalidade, apresenta uma dinâmica mais complexa que no caso com parâmetros constantes. Também foi observado que a variação sazonal da taxa de crescimento intrínseco inclui o estágio de dormência da broca da cana, em determinado período do ano. No segundo modelo apresentado, que descreve as interações dessas três populações, consideramos os efeitos da sazonalidade e é possível observar que o sistema também passou a exibir dinâmica caótica.

Portanto, espera-se que esse estudo auxilie na compreensão da dinâmica sazonal da intereção da broca da cana-de-açúcar e seus parasitoides. Uma vez conhecida essa dinâmica, como continuação desse trabalho será aplicado o controle biológico da broca da cana-de-açúcar, utilizando os dois parasitoides.

\section{Agradecimentos}

O primeiro autor agradece à CAPES pelo auxílio financeiro na realização do trabalho.

\section{Referências}

[1] Bezerra J.I.M., Molter A., Rafikov M. and Frighetto D.F. Biological control of the chaotic sugarcane borer-parasitoid agroecosystem. Ecological Modelling, 2021. DOI: 10.1016/j.ecolmodel.2021.109564.

[2] Garcia, J.F., Grisoto, E., Botelho, P.S.M., Parra, J.R.P. and Appezzato-Da-Glória, B. Feeding site of the spittlebug Mahanarva fimbriolata (Stal) (Hemiptera: Cercopidae) on sugarcane. Scientia Agricola, 2007. DOI: 10.1590/S0103-90162007000500014

[3] Goh, B.S. Management and analysis of biological populations, Elsevier, New York, 1980.

[4] Molnár, S., López, I., Gámez, M. and Garay, J. A two-agent model applied to the biological control of the sugarcane borer (Diatraea saccharalis) by the egg parasitoid Trichogramma galloi and the larvae parasitoid Cotesia flavipes, Biosystems, 2016. DOI: 10.1016/j.biosystems.2016.02.002

[5] Rafikov, M. and Limeira, E.H. Mathematical modelling of the biological pestcontrol of the sugarcane borer. International Journal of Computer Mathematics, 2012. DOI: 10.1080/00207160.2011.587873.

[6] Rafikov, M. and Silveira, J.C. Dynamics of the sugarcane borer-egg parasitoid-larvae parasitoid agro-ecosystem. In: International Conference MPDE'13-Models in Population Dynamics and Ecology, University of Osnabrück, Germany, 26-29 August 2013, Abstracts, p. 57. 2013.

[7] Rafikov, M. e Silveira, J.C., On dynamical behavior of the sugarcane borer - Parasitoid agroecosystem, Ecological Complexity, 2014. DOI: 10.1016/j.ecocom.2013.12.003. 